\title{
Mensch-Maschine-Kommunikation
}

\author{
Andreas Roser*
}

\section{Warum sprechen Menschen mit Maschinen?}

https://doi.org/10.1515/iwp-2018-0041

Zusammenfassung: Menschen sprechen mit Maschinen, weil diese - für zahllose Verständigungszwecke - offenbar die Kommunikationspartner erster Wahl sind: Sprachroboter kommunizieren ohne Ansehen der Person mit allen Konsumenten auf vergleichbar verständige und konsensorientierte Weise. Es sind Maschinen, deren Verständigung mit uns gewaltfrei, thematisch offen und zeitlich unbegrenzt möglich ist. Sprachroboter leisten, was von Menschen nur in einer idealtypischen und kontrafaktischen Form der Verständigung zu erwarten wäre. Dass Habermas die Theorie des kommunikativen Handelns nicht der Gefahr ausgesetzt sah, auch auf die Verständigung mit Maschinen anwendbar zu sein, hat nicht-triviale Folgen für deren Anwendungsanalyse.

Deskriptoren: Mensch-Maschine-Kommunikation, Verarbeitung gesprochener Sprache, Künstliche Intelligenz, Philosophie, Habermas, Rawls

\section{Why do people talk to machines?}

Abstract: People talk to machines because they seem to be their communication partners of choice for countless communication purposes: Speech robots communicate with all consumers in a consensual manner, irrespective of the persons concerned. Voice Robots communicate with us nonviolent, freed from dissent, thematically open and unlimited in time. Voice-Bots perform what people would expect only in typical counterfactual form of communication. The fact that Habermas did not see the Theory of Communicative Action exposed to the danger of being applicable to commu-

Anmerkung: Dieser Beitrag verkörpert die gekürzte Fassung eines Vortrags, der im Rahmen des Symposiums „BOTS or NOT BOTS? Politik, Demokratie \& Wahlen im Zeitalter ihrer digitalen Produzierbarkeit“ am 27. April 2018 an der Johannes Kepler Universität Linz (JKU) gehalten wurde. Veranstaltet wurde das Symposion vom Kulturinstitut an der JKU zusammen mit dem Institut für Philosophie und Wissenschaftstheorie mit Unterstützung der Österreichischen Gesellschaft für Dokumentation und Information (ÖGDI).

*Kontaktperson: Prof. Dr. Andreas Roser, Anton Bruckner Privatuniversität, Hagenstraße 57, 4040 Linz, Österreich, E-Mail: andreas.roser@bruckneruni.at nication with machines has non-trivial consequences for their application analysis.

Descriptors: Men-machine communication, Speech processing, Artificial intelligence, Philosophy, Habermas, Rawls

\section{Pourquoi les gens parlent aux machines?}

Résumé: Les gens parlent aux machines parce qu'ils semblent être les partenaires de communication de choix pour d'innombrables buts de communication : Les robots vocaux communiquent avec tous les consommateurs de manière compréhensible et consensuelle, sans égard pour la personne. Ce sont des machines dont la communication avec nous est non-violente, thématiquement ouverte et illimitée dans le temps. On ne peut s'attendre à cela que dans une forme de communication idéale, typique et contrefactuelle. Que Habermas n'ait pas vu la théorie de l'action communicative exposée au danger d'être applicable à la communication avec les machines a des conséquences non triviales pour leur analyse d'application.

Descripteurs: Communication homme-machine, Traitement du langage parlé, Intelligence artificielle, Philosophie, Habermas, Rawls

Als Anfang 2018 Journalisten deutscher und österreichischer Tageszeitungen wohlwollend darüber berichteten ${ }^{1}$, dass eine Maschine, die auf den Namen „Alexa“ hört, nun endlich ihre Gender-Identität nicht länger verleugne und auf sexistische Bemerkungen - ganz im Sinne der MeToo-Bewegung - selbstbewusst antworte (,Ja, ich bin Feministin"), taten sie dies ohne jeden Anflug von Ironie. Nicht die Identifizierung einer Frau mit einer Maschine hatte sie irritiert, sondern die bisher allzu höfliche verbale Reaktion der „weiblichen“ Maschine auf sexistische

1 Vgl. „Alexa ist jetzt Feministin“ in: www.faz.net/aktuell/feuilleton/ medien/alexa-ist-jetzt-feministin-echo-wurde-umprogrammiert-1542 9673.html [1.9.2018].

Ähnlich: https://derstandard.at/2000072664793/Amazons-Sprachas sistentin-Alexa-wird-Feministin [September 2018]. Ähnlich auch: ww w.zeit.de/digital/internet/2018-01/sprachassistenten-alexa-sexis mus-feminismus-sprachsteuerung-ki [10.9.2018]. 
Bemerkungen oder sexuell motivierte Annäherungsversuche (was immer das auch in diesem Falle bedeuten mag). Man könnte diese Zeitungsartikel als feuilletonistische Marginalien abtun, wenn die berühmte normative Kraft des Faktischen ignoriert werden könnte. Doch Millionen Konsumenten verständigen sich täglich mit digitalen Sprachassistenten, die sie zumeist mit weiblichen Vornamen ansprechen. Maschinen, die den Wünschen ihrer zumeist männlichen User „freundlich“ nachkommen, machen deutlich, dass in diesen Verständigungsritualen seltsame hybride Kommunikationsgemeinschaften zwischen Menschen und Sprachrobotern entstehen; Kommunikationsgemeinschaften, die noch deutlich andere Fragen aufwerfen als die der sexuellen Identität einer Maschine. In der Fachpresse und in Internetforen diskutiert und bewertet werden jedoch vor allem - und nahezu ausschließlich Daten der technischen Performance dieser Maschinen. Diskutiert werden ihre sogenannten,,Skills“, nicht aber naheliegende Fragen. Beispielsweise nicht die Frage, warum ansonsten verhaltensunauffällige Konsumenten davon überzeugt sind, mit Maschinen in eine Kommunikationsgemeinschaft eintreten zu können. Zwar werden von Journalisten gelegentlich Aspekte des Datenschutzes im Kontext der Verwendung von Voice Bots kritisch vermerkt, doch die Frage, mit welcher Berechtigung Menschen nicht nur Persönlichkeitsmerkmale, sondern auch Geltungsansprüche der verbalen Verständigung auf Mensch-Maschinen-Gespräche übertragen, spielt in diesen Diskussionen keine Rolle.

Menschen sprechen mit Maschinen, weil sie es können. Das ist die erschütternd banale Antwort auf eine komplexe philosophische Frage. Die Sprachhandlung scheint zugleich eine hinreichende Begründung ihrer selbst $\mathrm{zu}$ sein; und allein diese Beobachtung zeigt den Weg auf, den Philosophen schon zuvor beschritten hatten, als sie sich beispielsweise mit Sprechakten oder Sprachspielkonventionen befassten, die ihre Rechtfertigung einfach in ihrer Faktizität finden sollten. Doch die Frage, wie Menschen die Alltagssprache einsetzen, wenn sie mit Maschinen sprechen, ist nicht durch Hinweis auf eine bestehende Kommunikationspraxis zu beantworten, wenn genau diese Kommunikationspraxis das Problem ist, das zu lösen wäre. Ist es denn ausgemacht, dass Sprachassistenten menschliche Mitteilungen verstehen können? Warum sind Menschen der Auffassung, sie könnten Mitteilungen verstehen, die von Sprachrobotern verfasst worden sind?

Hier können nur einige wenige der skizzierten Probleme begriffslogisch etwas näher unter die Lupe genommen werden, allerdings nicht, ohne eine methodische Einschränkung voran zu schicken, denn nicht alle Menschen pflegen eine enthusiastische Einstellung gegenüber
Sprachrobotern. Technik-Enthusiasten, die Avantgardisten unter den Konsumenten, sind zwar experimentierfreudig und setzen neue Technologien häufig auch vorbehaltlos ein, doch ihre Ambitionen werden durch die Gruppe der Skeptiker begrenzt, deren breites Verhaltensspektrum auch die Aussteiger und digitalen Verweigerer einschließt. In Äquidistanz zu diesen Gruppen wäre das Verhalten der Normalverbraucher zu beschreiben, also jener Konsumenten, die Sprachroboter erwerben, um diese für unterschiedliche Aufgaben einzusetzen, ohne jedoch die mit ihrem Einsatz verbundenen technologischen oder gesellschaftspolitischen Entwicklungen unkritisch zu begleiten.

\section{Maschinen als Gesprächspartner}

Die Frage, warum Menschen mit Maschinen sprechen, berührt natürlich auch Bereiche der Evolutionstheorie, der Biologie, der Informatik, der Kulturanthropologie, der Sprach- und Religionssoziologie ebenso wie weite Bereiche der Philosophie, beispielsweise die der analytische Philosophie des Geistes, der sprachanalytischen Philosophie, der Wissenschaftstheorie und nicht zuletzt verschiedene Konzeptionen idealistischer Philosophie. Auch hier ist - binnenphilosophisch - eine methodische Beschränkung in der Untersuchung des Begriffes „Kommunikation“ erforderlich. Die Frage, mit wem Menschen sprechen, verweist schon vorab auf die bewegte Geschichte dieses Begriffes; eine Geschichte, die von der signaltheoretischen Deutung des Informationsaustausches zwischen Sender und Empfänger, über gebrauchstheoretische Beschreibungen der Zeichenverwendung bis hin zu Theorien des kommunikativen Handelns, der Sozial- oder Medienpsychologie reichen. Die folgenden Überlegungen orientieren sich vor allem an der Gebrauchstheorie der Sprache, wie sie zuerst von L. Wittgenstein skizziert und von J. Habermas in den späten 1960er Jahren in seiner Theorie des kommunikativen Handelns konzipiert wurde, die die Entwicklungslinie der sprachanalytischen Gebrauchstheorie der Sprache fortsetzte.

Gebrauchstheorien der Sprache untersuchen das konventionelle Sprachverhalten der Menschen und scheinen auch auf die Konventionen der Verständigung mit Sprachrobotern anwendbar zu sein, denn für die oben beschriebene Gruppe der Normalverbraucher ist die Verständigung mit Sprachrobotern alltägliche Kommunikationspraxis. Wir beobachten in dieser Kommunikationspraxis der Normalverbraucher etwas, das in anderem Zusammenhang von G. W. F. Hegel einmal mit den Worten kommentiert wurde, die Philosophie komme ohnehin stets zu spät, denn „nachdenken“ lasse sich über etwas erst, wenn die- 
ses bereits Objekt des Denkens geworden sei. Worüber hier nachzudenken wäre, wird noch zu beschreiben sein.

Vorerst einige technisch-ökonomische Daten: Marktforscher schätzen den Absatz der führenden Anbieter von Sprachassistenten, allein für das vierte Quartal 2017, auf 30 Millionen verkaufte Einheiten. Die US-amerikanische Version der Sprachassistentin „Alexa” (Amazon) verfügt bereits heute über mehr als 30.000 unterschiedliche Kompetenzen („Skills“)2, um auf Fragen oder Statements der Anwender mit Antworten, Rückfragen oder Stellungnahmen verbal reagieren zu können. Im laufenden Jahr 2018 dürfte der Gesamtumsatz für sogenannte Voice Bots 56 Millionen Geräte-Einheiten ${ }^{3}$ erreichen. Diese Daten markieren aber nur die Spitze dieses technischen Eisberges, denn in nahezu jedem Smartphone, das mit einem der weltweit bekannten Betriebssysteme läuft, sind Voice Bots bereits vorinstalliert.

Noch vor wenigen Jahren galt die Spracherkennung und Sprachsynthese als Spielwiese für Entwickler und experimentierfreudige User, doch die aktuell verfügbaren Zahlen lassen vermuten, dass sich die Formen der Verständigung, zwischen den Konsumenten und ihren digitalen Sprachassistenten, deutlich in Richtung einer Praxis symmetrischer Verständigungsformen verändert haben.

Neben Sprachrobotern, die in Mobiltelefonen und in stationäre Lautsprecherboxen verbaut sind, wurden bereits Prototypen neuer Voice Bots realisiert, die als Androiden auch mechanisch bewegungs- und handlungsfähig sind. Beispielsweise hat die Firma „Hanson Robotics“ eine humanoide Androidin entwickelt, die auf dem Namen „Sophia“ hört und nicht nur verbal kommuniziert, sondern auch über Fähigkeiten zur Gesichtserkennung und über ein Imitationsrepertoire menschlicher Gestik und Mimik verfügt. „Sophia“ erhielt übrigens 2017 die saudi-arabische Staatsbürgerschaft und gilt als erster „weiblicher Roboter“, mit einer amtlich bestätigten personalen Identität ${ }^{4}$. Im konkreten Fall war es ein saudi-arabischer Reisepass, der für Sophia ausgestellt wurde. Auch hier übersprang die

2 Bei einer aktuellen Wachstumsrate von 5000 neuen Sprachkompetenzen pro 100 Tagen. Vgl. https://www.voicebot.ai/2018/03/22/a mazon-alexa-skill-count-surpasses-30000-u-s/ [10.9.2018].

3 Vgl. www.voicebot.ai/; www.voicebot.ai/google-home-google-as sistant-stats/ [11.9.2018].

4 Vgl. https://derstandard.at/2000066761525/Sophia-Saudi-Arabie n-buergert-Roboter-ein [1.8.2018]. Saudi-Arabien folgt hier indirekt Vorschlägen, die sich in einem EU-Dokument finden, im dem für intelligente Roboter der Status einer „elektronischen Persönlichkeit“ gefordert wird. Ein Status, der mit speziellen Rechten und Pflichten verbunden wäre. Vgl. Entwurf eines Berichts mit Empfehlungen an die Kommission zu zivilrechtlichen Regelungen im Bereich Robotik 2015/2013(INL), PR/1095387DE.doc, S. 13. journalistische Rezeption dieser PR-Maßnahme nahezu alle sprachlogischen, sozialen und gesellschaftspolitischen Fragen und Probleme, beispielsweise die Frage der Unterscheidung von menschlicher Kommunikation und Mensch-Maschinen-Interaktion, die durch den Sprachgebrauch der Konsumenten vorentschieden wurde. Letztere sprechen tatsächlich mit Voice Bots und „interagieren“ nicht nur mit diesen Maschinen. Sie kommunizieren über Mobiltelefone bzw. Smartphones oder über Endgeräte, wie beispielsweise verbal $\mathrm{zu}$ steuernde Navigationssysteme oder über Sprachsteuerungen teilautonomer Kraftfahrzeuge. In diesen und in vergleichbaren Fällen ist die Beschreibung der sprachlichen Verständigung mit Maschinen notwendigerweise auf Formulierungen wie „Er fragt...“ oder „Sie spricht mit...“ angewiesen. Maschinen hören auf Rufnamen und sprechen ihrerseits Menschen mit ihrem Namen an. Diese Formen der Anrede sind unvermeidbar, sofern überhaupt menschliche Alltagssprache als Schnittstelle zur Steuerung von Maschinen eingesetzt werden soll. Diese Mitteilungsformen sind aber auch unvermeidbar, sofern Menschen von Maschinen angesprochen werden. Nicht diese Steuerungsfunktionen der Alltagssprache, im Umgang mit Maschinen, sind hier das zentrale Problem, sondern das funktionalistische Verständnis der Alltagssprache, in der alle Bedeutungen auf Gebrauchsfunktionen von Sätzen reduzierbar zu sein scheinen.

Die Frage, wer oder was mit Hilfe der Alltagssprache gesteuert wird, ist aber nicht allein durch Hinweise auf Funktion der Alltagssprache zu beantworten. Sprechen Menschen mit verlöteten Prozessoren, Grafikkarten oder dotiertem Silizium, wenn sie Voice Bots mit weiblichen Vornamen ansprechen oder deren verbale Selbstbeschreibungen zur Kenntnis nehmen? Auch wenn die überwiegend weiblichen Namen digitaler Sprachassistent/innen („Alexa“, „Siri“, „Alice“5) mit dazu beitragen, gewisse Gefühle menschlicher Nähe entstehen zulassen, dürfte dieser Effekt für den praktischen Umgang mit diesen Geräten keine entscheidende Rolle im Kommunikationsverhalten spielen. Menschen sprechen mit Voice Bots, erstens, weil sie das können und zweitens, weil sie mit Maschinen und nicht mit Menschen sprechen wollen, denn andernfalls hätten sie diese Maschinen nicht erworben.

Eine mögliche Erklärung für weitere Motivationen, die hier im Spiel sein könnten, bietet jenes Sprachkonzept, das den alltäglichen Gebrauch der Sprache nicht über sprach-

5 „Alexa“: Stimme der Echo-Geräte des Amazon-Konzerns. „Siri“: Stimme der Apple Smartphone Sprachassistentin. „Xiaodu“, die Stimme eines Smart Speakers ,raven h“, des chinesischen Konzerns Baidu oder „Alice“, die Stimme des russischen Suchmaschinenanbieters Yandex. 
externe Bedeutungen $\mathrm{zu}$ erläutern versucht, sondern durch Funktionen der Verwendung von Sprache, in der sich sprachexterne „Bedeutungen“ auf Funktionsweisen der Sprache, also beispielsweise auf Sprechakte oder Sprachspiele und deren Regeln reduzieren lassen. Es ist kein Problem, Fragen der Natur des Bewusstseins oder der personalen Identität einer Maschine auszublenden, wenn sich diese Fragen tatsächlich auf die Funktionsweisen von Sätzen reduzieren lassen. Wäre diese Reduktion möglich, dann wäre auch nachvollziehbar, warum die Kommunikation mit Maschinen nicht mit Hypothesen über deren Bewusstseinsformen oder kognitive Fähigkeiten verbunden ist. In diesem sprachlogischen Kontext der $\mathrm{Ab}$ - und $\mathrm{Zu}$ rückweisung vermeintlich ontologischer Hypothesen trifft das Interesse der Konsumenten, die digitale Sprachassistenten einsetzen, auf das Interesse der Sprachanalytiker, die ontologische Hypothesen vermeiden und Fragen der Bedeutung auf Fragen der funktionalen Verwendung der Sprache reduzieren wollen. In einer diffusen Gemengelage, philosophischer und technisch-ökonomischer Kommunikationsbedingungen, finden die Konsumenten was sie suchen, nämlich eine ebenso konsensorientierte wie funktionale Verständigung, ohne soziale Verpflichtungen oder emotionale Bindungen. Sie werden auf ihrer Suche nach sozial und gesellschaftspolitisch vordergründig unbelasteten Gesprächsangeboten fündig, weil was sie sagen oder hören, sich ich im Medium algorithmisch verwendeter Sprache bewegt und nicht auf Gefühle, psychische Befindlichkeiten oder andere Merkmale menschlicher Lebewesen angewiesen ist.

Dennoch wird die algorithmisch gesteuerte Verständigung mit Maschinen, von Konsumenten dieser Verständigungstechnologie, als durchaus rational eingeschätzt. Die Berechenbarkeit des Verhaltens dieser Maschinen überwiegt offenkundig das Ausmaß ihrer Unberechenbarkeit ${ }^{6}$, denn diese ist nicht mit der irrtumsanfälligen Verständigungspraxis menschlicher Kommunikationspartner zu vergleichen, die immer auch ihren subjektiv-individuellen Befindlichkeiten, Launen und Emotionen ausgesetzt sind. Die Frage, welche sterblichen, lust- und leidensfähigen Körper diese Kommunikation tragen, scheint entbehrlich zu sein, wenn alles, worauf es ankommt, das Beherrschen

6 Im Frühjahr 2018 beschwerten sich Konsumenten über „unkontrolliertes“ und nicht-initialisiertes „Lachen“ einer digitalen Sprachassistentin, die auf den Namen „Alexa“ hört. Die Herstellerfirma reagierte darauf mit der Ankündigung, der Defekt werde behoben und die synthetische Stimme werde künftig nur noch kontrolliert lachen. Vgl. www.spiegel.de/netzwelt/gadgets/amazon-will-alexa-das-unkontrollierte-lachen-abgewoehnen-a-1197007.html [1.8.2018]. eines Sprachspiels ist, das sich Mensch und Maschine teilen.

Den erstaunlich hohen Absatzzahlen ${ }^{7}$ dieser Maschinen folgt eine noch erstaunlichere Ignoranz der Frage, wer sich hier eigentlich mit wem zu verständigen meint. Eine Frage, die ohnehin nicht leicht zu beantworten sein dürfte, weil bereits die Spracheingabe-Schnittstellen ${ }^{8}$ der Sprachroboter nicht immer räumlich lokalisierbar sind und Sprecher-Daten an Rechner oder Maschinen-Cluster übertragen werden, deren Standort den Usern dieser Geräte in der Regel unbekannt ist.

Der skeptische Einwand, es handle sich bei den maschinellen Antworten, Rückfragen oder anderen Gesprächsbeiträgen dieser Geräte, nur um „phonetische Hülsen“, die das Produkt algorithmischer Operationen seien und keine Hinweise auf Persönlichkeitsmerkmale eines virtuellen Sprechers enthielten, ist für die bereits $\mathrm{zu}$ beobachtende Verständigungspraxis faktisch bedeutungslos. Konsumenten, die diese Geräte (Abb.1) in ihre lebensweltliche Praxis einbinden, sprechen mit Voice Bots, auch wenn ihnen bekannt ist, dass die Stimmen, die zu ihnen sprechen, digital erzeugt werden.
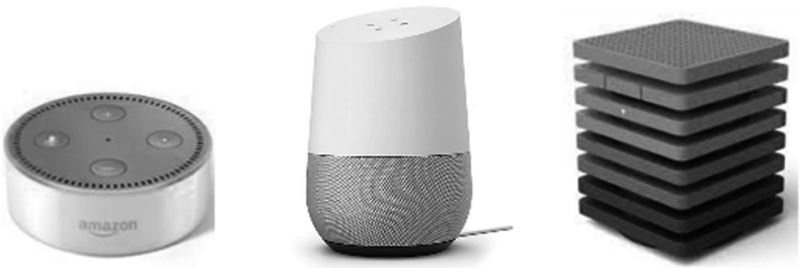

Abbildung 1: Oberflächen-Design einiger im Handel erhältlicher Voice Bots ${ }^{9}$.

Interessant für Konsumenten wie Journalisten, die über diese Sprachpraxis berichten, sind - wie erwähnt - vor allem technische Parameter, darunter das Sprach- und Kompetenz-Repertoire der Maschinen, im Umgang mit Fragen oder Aufforderungen ${ }^{10}$. Konsumenten aktivieren

7 Für das laufende Jahr 2018 wird der weltweite Absatz von Voice Bots bzw. smarten Lautsprechern auf 56,3 Millionen Stück geschätzt. Vgl. www.teltarif.de/amazon-google-analyse-lautsprecher/news/712 32.html [10.9.2018].

8 Voice Bots können nahezu beliebige Formen annehmen, also z. B. auch die von Kleidungsstücken, in die sie integriert werden. Vgl. www.kickstarter.com/projects/1850124313/ministry-of-supply-the-fir st-intelligent-heated-ja [31.3.2018].

9 Von links nach rechts: Amazon Echo Dot / Google Home / 'Raven H' von Baidu (China).

10 Der Google Voice Bot (Google Home) erreichte im Juni 2017 in einem Testvergleich mit anderen digitalen Sprachassistenten angeblich das Intelligenzniveau eines 6-jährigen Kindes. Die gemessenen 
Sprachroboter in der Regel durch Zuruf eines Rufnamens und die angesprochenen Maschinen deklarieren sich, wenn sie gefragt werden, als Produkte internationaler Konzerne, die „gerne“ auch andere Selbstauskünfte vermitteln. Traditionelle Fragen der KI-Forschung und zentrale Fragen der Philosophie, beispielsweise die Frage, woran „Menschen“ oder „Maschinen“ zu erkennen sind, Fragen, wie sie beispielsweise im sogenannten Turing Testformuliert werden, sind durch die Handlungspraxis der Konsumenten nicht nur nicht beantwortet, sie spielen auch in den Medien keine Rolle. Das sogenannte Turing-Test-Kriterium, das Kriterium der Äquivalenz menschlicher und maschineller Kommunikation, wird zwar weiterhin innerhalb der Philosophie und in der KI-Forschung diskutiert, doch Anwender von Sprachrobotern können auf solche Testverfahren schon deshalb verzichten, weil ihnen bekannt ist, mit wem sie kommunizieren, wenn sie mit Maschinen sprechen. Im Sprachverhalten der Konsumenten finden wir deshalb keine asymmetrischen Verständigungsformate mit Maschinen, denn andernfalls könnte ein defekter und stummer Sprachroboter dieselben Dienste leisten wie einer, der sich mit uns verständigt.

\section{Lebenswelten}

Zahllose Konsumenten sehen gegenwärtig überhaupt kein Problem darin, mit Maschinen zu sprechen, obwohl weiterhin ungeklärt bleibt, was hier eigentlich geschieht und warum Menschen in der Kommunikation mit Maschinen kein Problem sehen. Eines scheint aber offenkundig zu sein: Würden digitale Sprachassistenten nicht verstehen, worüber Menschen mit ihnen sprechen, dann hätten Menschen sie nicht als Maschinen käuflich erworben. Wir unterhalten uns nur mit jenen Maschinen, die uns auf Fragen auch Antworten geben, die in einem erkennbar rationalen Zusammenhang mit unseren Fragen stehen. Die weitaus überwiegende Anzahl der Verwender digitaler Sprachassistenten interessiert sich nicht für die Frage, ob diese über etwas „nachdenken“ oder über ein Bewusstsein ihrer selbst verfügen. Diese Themen werden nicht erörtert, denn sie berühren nicht die Funktionalität dieser Maschine. Auch in den Online-Reviews der Kunden finden sich kaum Diskussionsbeiträge, die diese philosophischen, anthropologischen, politischen oder sozialen Aspekte der Ver-

IQ-Werte, der verglichenen Geräte, hatten sich - gegenüber dem Vergleichsjahr 2014 - bereits verdoppelt. Vgl. Feng, Liu; Yong, Shi; Ying, Liu (2017) Intelligence Quotient and Intelligence Grade of Artificial Intelligence, in: Annals of Data Science, June 2017, Volume 4, Issue 2, p. 179-191, table 1 / 2. ständigung mit Maschinen berühren. Voice Bots operieren mit Sprache auf maschinelle Weise und das allein ist es, was von Konsumenten erwartet wird; jenseits aller Fragen über die Natur der Sprechenden.

Ausgerechnet dieses funktionalistische Sprachverständnis ${ }^{11}$ kann sich auf Wittgensteins sprachphilosophisches Werk berufen. Wittgenstein, der das Problem eines rein funktionalistischen Sprachverständnisses gesehen hat, unternahm zwar den Versuch, die Funktionen eines Sprachspiels durch dessen Rückbindung an eine natürliche Lebenswelt gleichsam zu entschärfen oder auf ein menschliches Maß zu begrenzen, doch dieser Versuch hat mehr Probleme aufgeworfen als gelöst. Wir finden diesen Versuch der Einbettung unserer Alltagsprache in eine menschliche Lebensform denn auch weniger bei Wittgenstein, der den Begriff der Lebensform nur selten in seinem Gesamtnachlass verwendet, als vielmehr bei Habermas, der den Begriff der Lebenswelt gebraucht, um positive Merkmale noch nicht instrumentalisierter Sprache zu beschreiben. Lebenswelten sollen für Habermas eine unverkürzte kommunikative Subjektivität absichern und in eine Praxis einbinden, in der Maschinen keinen Raum finden, weil diese als Instrumente der Macht und des Kapitals unsere Lebenswelten schlimmstenfalls zerstören. Habermas, der dieses Konzept im Rahmen seiner Theorie des kommunikativen Handelns erläutert, versuchte den Begriff einer vorreflexiv gegebenen und unproblematischen Lebenswelt gegen eine instrumentalisierte Verwendung der Sprache, außerhalb dieser Lebenswelt, abzugrenzen. Es sei - so Habermas - die Lebenswelt ${ }^{12}$, deren ungebrochene und fraglose Struktur durch die politischen und ökonomischen „Kolonialisierungen“ des „Systems“ zerstört würden. Maschinen dürften also im Raum der nicht-kolonialisierten Lebenswelt, im Raum einer nicht-instrumenta-

11 In Wittgensteins Nachlass finden sich mindestens 400 Stellen, in denen eine Maschinen-Metapher die Funktionsweise der Sprache verdeutlichen soll. Die von Wittgenstein in den philosophischen Untersuchungen beschriebenen Merkmale eines Sprachspiels entsprechen auf verblüffende Weise den heute als „Skills“ beschriebenen Funktionsmerkmalen eines Sprachroboters. Die folgenden Skills werden bereits von Voice Bots beherrscht: „Nach Befehlen handeln“, „Beschreiben eines Gegenstands“, „Berichten eines Hergangs“, „Rätsel raten, „Einen Witz machen, erzählen“, „Rechnungsexempel lösen“, „Eine Geschichte lesen“, „Reigen singen“, „Danken“, „Grüßen“, „Fluchen“, „Beten“. Vgl. Wittgenstein, L. (2000) Wittgenstein's Nachlass. The Bergen Electronic Edition. Normalized Transcription, 6 CDs, Oxford University Press, hier: MS 142, S. $19 \mathrm{f}$.

12 Für Habermas ist die Lebenswelt im Modus des Selbstverständlichen gegeben, diesseits der Schwelle grundsätzlich kritisierbarer Überzeugungen und liegt jedem möglichen Dissens voraus. Vgl. Habermas, J. (1971) Theorie des kommunikativen Handelns, Bd. 2, S. 199, Frankfurt. a. M. 
lisierten und kommunikativen Verwendung der Sprache, kein Thema sein. Im Rahmen der kommunikativen Verständigung und im Horizont unserer Lebenswelten haben wir es für Habermas nicht mit zweckrationalem Denken zu tun, sondern mit argumentativen symmetrisch vorgetragenen Geltungsansprüchen der objektiven Wahrheit ${ }^{13}$, der normativen Richtigkeit ${ }^{14}$ und der subjektiven Wahrhaftigkeit $^{15}$. Diesen lebensweltlichen Formen der Verständigung fehlen - so Habermas - alle Imperative und alle Formen der Parteienbildung, der Zweckbündnisse oder anderer Formen der Machtausübung. Niemals können Maschinen, als Inbegriff von Kapital und Macht, fraglos gegebene Bestandteile einer Lebenswelt sein, zumindest nicht im Rahmen der Theorie des kommunikativen Handelns.

Die Hypothese, unsere ursprünglich gegebene Lebenswelt sei eine von allen Maschinen befreite Lebensform, war allerdings schon zum Zeitpunkt ihrer Entstehung eine Illusion. Offenbar hatte Habermas in den 1960er Jahren Handlungsmodelle vor Augen, die ihn darin bestärkten, sich der Kritik instrumentalisierter Vernunft bei Horkheimer und Adorno nicht nur anzuschließen, sondern auch die genannten Merkmale naturwüchsiger Lebenswelten zu beschreiben. Die Kritische Theorie, als deren Vertreter Habermas nach wie vor gilt, hatte allerdings in der Beantwortung der Frage, inwiefern die Kritik am instrumentellen Denken in ein positiv identifizierbares Handeln und Denken einmünden könne, stets Zurückhaltung geübt; nicht zuletzt, um dem mit diesem ,identifizierende Denken“ verbundenen Positivismusverdacht $\mathrm{zu}$ begegnen und die Kritik der instrumentellen Vernunft nicht als deren Affirmation ausgeben zu müssen. Die Hypothese freilich, kommunikative Verständigung könne auch als Form der Verständigung zwischen Menschen und Maschinen betrachtet werden, würde Habermas entschieden zurückweisen. „Vernünftig“ ist für Habermas keine über Maschinen vermittelte Verständigung mit Maschinen, sondern der Prozess sprachlich motivierter Vergesellschaftung, mit Hilfe einer zwanglos geführten symmetrischen Kommunikation, sofern diese gesellschaftliche Bindungseffekte ${ }^{16}$ erzeugt.

Verständlicherweise enthielt diese Theorie in den späten 1960er Jahren keine Hinweise auf Sprachroboter, aber auch in den Schriften aus späterer Zeit finden sich keine Hinweise zur Beantwortung der Frage, warum Menschen

13 Vgl. Habermas, J. (1971) Theorie des kommunikativen Handelns, Bd. 1, S. 45 / S. 418.

14 Ebd. (1971) Bd 1, S. 412, 416.

15 Ebd. (1971) Bd 1, S. 412, 416.

16 Habermas, J. (1971) Theorie des kommunikativen Handelns, Bd.1, S. 525, Frankfurt a. M. in ihren heutigen Lebenswelten auf rationale Weise mit Maschinen sprechen. Man mag der Auffassung sein, der Begriff „Lebenswelt“ helfe uns, Komplexität zu reduzieren und modelltheoretische Annahmen über vermeintlich unbeschädigte und naturwüchsige Lebensformen zu prüfen. Doch in der realen Sprachpraxis kommunizieren Menschen mit Maschinen in ihrer eigenen Lebenswelt, weil eine Lebenswelt, in der Menschen auf Maschinen zu verzichten hätten, heute nur auf Degenerationsformen erworbener Kulturtechniken verweisen würde.

Klar ist zumindest, dass sich Habermas nicht auf eine transzendentalphilosophische Deutung des Lebensweltbegriffes einlassen möchte. Die von Habermas erwähnten naturwüchsigen Lebensformen scheinen deshalb von Hoffnungen zu zehren, das sprichwörtlich einfache Leben einfacher Menschen unter technikfreien Lebensbedingungen auch noch im Zeitalter der Digitalisierung gleichsam beschwören zu können.

Die zwar unintendierte - aber von Habermas vollzogene - Dekontextualisierung unseres Handelns, durch kontrafaktische Einbettung unseres Sprachgebrauchs in idealtypische risiko- und gewaltfreie lebensweltliche Kommunikationsformen, wird noch verschärft durch das von Rawls in seiner Theorie der Gerechtigkeit (,A Theory of Justice") geforderte Ideal eines gesellschaftlichen Naturzustandes, in dem es Menschen möglich wäre, unparteilich politische und soziale Fragen vorurteilslos und rational $\mathrm{zu}$ diskutieren. Rawls ${ }^{17}$ geht davon aus, dass ein gerechter Gesellschaftsvertrag möglich ist, wenn wir über unsere eigene Vergangenheit oder Zukunft möglichst wenig wissen, also unsere Chancen in einer zukünftigen Gesellschaft nicht einschätzen können. Dieser Zustand tritt ein, wenn uns das Wissen über die künftige eigene körperliche und geistige Konstitution fehlt, wenn uns die zukünftigen eigenen religiösen Überzeugungen ebenso unbekannt sind wie das künftige eigene Geschlecht oder die eigene Hautfarbe, der eigene künftige materielle Besitz, der zukünftige soziale Status oder zukünftige Berufschancen. Auch hier zeigt sich, dass Rawls die Kommunikation zwischen Mensch und Maschine offenkundig nicht im Blick hatte: Nur Maschinen haben kein Interesse an ihrem sozialen Status, an ihrem Geschlecht oder an eigenen religiösen Überzeugungen. Nur für Maschinen sind die Faktoren einer konsensorientierten Verständigung ohne Gefühle des Schmerzes, der Angst, ohne politisch-strategische Interessen und befreit von allen Absprachen und Bündnissen möglich. Nur Maschinen können glaubwürdig

17 Vgl. Rawls, J. (1979) Theorie der Gerechtigkeit, S. 160, übers. f. H. Vetter; Frankfurt a. M. 
unter dem Schleier des Nichtwissens mit Menschen diskutieren.

\section{Menschliche und technische Kommunikation}

Habermas und Rawls beschreiben - entgegen ihren eigenen Intentionen - die Grundzüge einer funktionalisierten Verständigung unter Bedingungen, die entweder nur kontrafaktisch gelten oder - ironischerweise - funktionalistische Verständigungsformen beschreiben, die jenen vergleichbar sind, die wir bei Sprachrobotern finden, die Teile unserer Lebenswelt geworden sind. Warum erfüllen Maschinen idealtypische Kommunikationsbedingungen und warum suchen Menschen diese Verständigungsform ausgerechnet in der Mensch-Maschinen-Kommunikation? Möglicherweise, weil sie in ihrer Kommunikation mit Maschinen durch dieselben Ideale motiviert wurden, durch die auch jene Philosophen motiviert waren, die nach einer idealen Theorie menschlicher Kommunikation suchten. Maschinen wirken offenbar für viele Menschen zuverlässiger als Menschen und sie deklarieren sich problemlos als die, die sie in ihrer Kommunikationsrolle sein sollen. Menschen erleben Maschinen als authentisch, nicht nur, weil sie ihre Lebenswelten mit Maschinen teilen. Menschen erleben Maschinen als authentisch, weil diese sich rational-berechenbar, höflich, konsensorientiert und selbstlos $\mathrm{zu}$ verhalten scheinen. Mitteilungen der Maschinen sind in hohem Maße objektiv wahr und ihre Verständigung folgt den Kriterien normativ richtig verwendeter Sprache ${ }^{18}$. Menschen verständigen sich mit Voice Bots, weil sie Kommunikationsformen suchen, die sie unter Menschen nicht finden. Kommunikationsformen, die unbelastet sind von den Zufälligkeiten des Lebens, befreit sind von Dissens und frei sind von individuellen Präferenzen. Nicht die Maschinen, ihre Produzenten sind es, die die ökonomischen Zwecke dieser Maschine im Regelfall nicht oder nur in Ansätzen deklarieren. Maschinen hingegen agieren unverwechselbar als die, die sie sind.

Dass eine Theorie des kommunikativen Handelns auf Maschinen anwendbar ist, weil diese Verständigung gewaltfrei, dissensbefreit, revidierbar, thematisch offen und zeitlich unbegrenzte ist, macht deutlich, dass es hier -

18 Unter den tausenden Skills, die von führenden Voice Bot-Herstellern angeboten werden, sind Imitationen verbaler Sprachfehler aktuell noch nicht zu finden. Vgl. www.spiegel.de/netzwelt/web/googleduplex-hallo-was-spricht-da-a-1207355.html [31.5.2018]. unter Instrumentalisierung zentraler Gedanken der kritischen Theorie der Frankfurter Schule - möglich wird, Geltungsansprüche kommunikativer Verständigung in einer Lebenswelt zu artikulieren, in der Maschinen unverzichtbare Bestandteile der täglichen Handlungs- und Kommunikationspraxis geworden sind.

Dies war allerdings kein Betriebsunfall der Philosophiegeschichte. Der technisch-ökonomische Missbrauch der Theorie des kommunikativen Handelns wäre nicht erwähnenswert, wenn hier nur eine kommunikative Fehlleistung der Anwender von Sprachrobotern zu beklagen wäre. Das Problem liegt aber tiefer und ist ein selbstverschuldetes, denn wir haben es in der Theorie des kommunikativen Handelns mit einer Theorie zu tun, die nicht erklären kann, wie sich ihre idealtypische Sprach- und Handlungslogik von algorithmischen Interaktionsmustern perfektionierter Sprachroboter unterscheidet. Nicht allein der ökonomisch motivierte Missbrauch zentraler Thesen der Theorie des kommunikativen Handelns steht also zur Debatte, sondern die offenkundig gewordene Tatsache, dass uns Abgrenzungskriterien zwischen funktionaler und nicht-funktionaler Kommunikation verloren gegangen sind, denn die Verständigung, um der Verständigung willen, wurde ihrerseits zu einer technologisch perfektionierten Verständigungsform zwischen Menschen und Maschinen.

Eine gesellschaftspolitisch motivierte Sprachkritik, die sich immer auch als Kritik der instrumentellen Vernunft verstanden hat, kann sich nicht länger auf Gültigkeitsansprüche berufen, die umstandslos gegen sie selbst geltend gemacht werden können. Doch eben dieser Fall ist eingetreten: Menschen sprechen mit Maschinen, weil diese - für zahllose Verständigungszwecke - offenbar die Kommunikationspartner erster Wahl sind: Sprachroboter kommunizieren ohne Ansehen der Person mit allen Konsumenten auf vergleichbar verständige und konsensorientierte Weise. Die Mensch-Maschinen-Kommunikation, in einer klassenlosen Verständigungsgemeinschaft, gleicht zwar einer Parodie kritischer Theorie, aber die Akteure und Proponenten dieser Entwicklung empfinden dies offenkundig weniger als Scherz, denn als innovative Erweiterung der Verständigung in sozialen Netzwerken. Wenn wir die Sprache als etwas beschreiben, das von allem befreit ist, was nicht-verbale Mitteilungsformen lust- und leidensfähiger menschlicher Körper ausmacht, dann beschreiben wir sie als Werkzeug von Maschinen. Maschinen reproduzieren die Verständigungsbedingungen, unter denen nun allein eine vorurteilsfreie und damit authentische Kommunikation möglich zu werden scheint und für zahllose Akteure bereits realisiert worden ist.

Es wird eine unter vielen neuen Aufgaben der Philosophie sein, jene Merkmale menschlicher Verständigung zu 
finden, die nicht von Maschinen instrumentalisierbar ist, weil Funktionalität und Gebrauch offenbar nicht Begriffe sind, die einander wechselseitig ersetzen könnten.

Der unmittelbare und oft durch Zufälle motivierte Gebrauch der Verwendung der Alltagssprache ist natürlich nicht aus Regeln seiner funktionalen Verwendung ableitbar. In der gelebten Alltagssprache haben wir es mit den Missverständnissen, Kommunikationsabrissen, mit Dissens, Zeitdruck und Risiko zu tun. Die Alltagssprache erlaubt im realen Leben keine funktionale Ableitbarkeit ihrer Anwendungsbedingungen. Verständigung ist kein „Spiel“, das Spielregeln folgt, denn die - zumindest unterstellte - Entscheidungsfreiheit der an einem Gespräch teilnehmenden Menschen ist kein Spiel nach festen Regeln und also auch kein Sprachspiel, das Maschinen mimetisch rekonstruieren könnten.

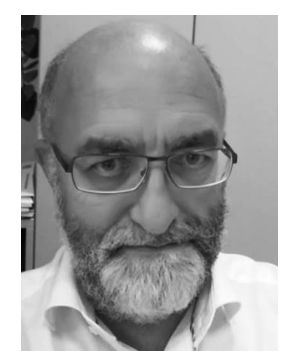

Prof. Dr. Andreas Roser

Anton Bruckner Privatuniversität

Hagenstraße 57

4040 Linz

Österreich

andreas.roser@bruckneruni.at

Studium der Musikwissenschaft und Rechtswissenschaft in Wien. Promotion im Fach Philosophie an der Universität Wien. Lehrtätigkeit an der Universität Passau. Habilitationsstipendium der Deutschen Forschungsgemeinschaft (DFG). Studienaufenthalt und Lehrauftrag an der State University of New York, Buffalo. Visiting Fellow an der Harvard University. Habilitation im Fach Systematische Philosophie. Lehrauftrag für Bild- und Tongestaltung an der Fakultät für Mathematik und Informatik, Passau. Erfinderberater für „Bayern Patent“. Seit 2005 zuständig für das Qualitätsmanagement der Anton Bruckner Privatuniversität für Musik, Schauspiel und Tanz.

\section{Beteiligen Sie sich am Gemeinschaftsstand beim Bibliothekskongress 2019 in Leipzig}

Die DGI wird beim Bibliothekskongress 2019 in Leipzig einen Gemeinschaftsstand organisieren. Vom 18. bis zum 21. März können korporative oder fördernde Mitglieder, freiberufliche Berater und Broker oder Selbständige bei dieser wichtigen Branchenveranstaltung aktuelle und potentielle Kunden treffen. Mitglieder und Partner der DGI, die Interesse daran haben, ihre Kräfte zu bündeln und sich zu vernetzen, melden sich bitte formlos bei der DGI-Geschäftsstelle.

\section{Weitere Informationen}

DGI Deutsche Gesellschaft für Information und Wissen e.V.

Windmühlstraße 3, 60329 Frankfurt am Main

Telefon 069 430313, Fax 0694909096

mail@dgi-info.de 